\title{
Elastic immersive wave experimentation: Theory and physical implementation
}

\author{
Henrik R. Thomsen $\odot,{ }^{*}$ Miguel Molerón, Thomas Haag, Dirk-Jan van Manen, and Johan O. A. Robertsson \\ Institute of Geophysics, ETH Zürich, 8092 Zürich, Switzerland
}

(Received 29 July 2019; published 26 December 2019)

\begin{abstract}
We present a proof of concept of elastic immersive wave experimentation. A physical experiment of finite volume is connected with a numerical domain via the novel theory of immersive boundary conditions. We show that by applying the incident traction measured at the free surface of a solid target, we can completely cancel unwanted boundary reflections in the physical domain. The propagating waves can then seamlessly interact with a virtual, numerical domain while we fully account for long-range interactions between the two domains. Utilizing a laser Doppler vibrometer, we can accurately record the three-component particle motion of the wave field at the surface of a thin aluminum beam. The recordings are used to iteratively construct the immersive boundary conditions which are applied to the lateral ends of the beam by three-component piezoelectric actuators. Our one-dimensional experimental results show that we can actively cancel the waves reflected at the free-surface end of the aluminum beam for individually excited, broadband longitudinal and flexural wave modes, as well as for the simultaneous excitation of the two. Finally, we introduce interactions between the physical and a desired numerical domain, thereby virtually extending the physical aluminum beam.
\end{abstract}

DOI: 10.1103/PhysRevResearch.1.033203

\section{INTRODUCTION}

Recently, a new paradigm for wave propagation experimentation, referred to as immersive wave experimentation (IWE), was proposed by Vasmel et al. [1]. This novel idea is based on the principle of immersive boundary conditions (IBCs) [2], which links a physical propagation domain of limited size to a virtual, numerical environment using an array of sensors and actuators situated on the boundaries of the physical experiment [3]. The outgoing waves are measured inside the physical domain and extrapolated to the boundaries. Here, the reflected waves are canceled and the interaction of the outgoing waves with the numerical environment computed and injected back into the physical domain. The physical boundaries of the solid target are thus made transparent to the propagating wave.

This new approach provides several potentially important novelties over conventional, purely physical laboratories. For instance, the possibility to completely remove unwanted boundary reflections enables investigation of a wide range of methods which require the data to be free of waves stemming from more than one interaction with the free surface. Such methods include experimental time reversal [4], Marchenko focusing [5], and full wave-form inversion [6]. Furthermore, IWE alleviates the necessity of downscaling the samples under study, in turn reducing assumptions about frequency

\footnotetext{
*henrik.thomsen@erdw.ethz.ch

Published by the American Physical Society under the terms of the Creative Commons Attribution 4.0 International license. Further distribution of this work must maintain attribution to the author(s) and the published article's title, journal citation, and DOI.
}

dependent wave phenomena, such as attenuation or dispersion [7-9]. Finally, the possibility to implement virtually any kind of boundary conditions, or virtual domains with arbitrary mechanical or acoustic properties (e.g., media with gain or negative constitutive parameters over a broad frequency range), offers new possibilities in the emerging fields of metamaterials [10,11], parity-time symmetry [12], as well as holography and cloaking [13,14]. Especially in these fields, the possibility to circumvent the difficulty of building materials with the desired properties will make practical realizations of theoretical proposals possible.

In the first, one-dimensional, acoustic implementation of IWE, presented by Becker et al. [3], the propagating wave fields are recorded inside a sound tube. These recordings are then forward extrapolated to the ends of the tube in real time, where they are reinjected to cancel any reflections. Compared to its acoustic implementation, elastic IWE involves additional challenges. For instance, elastic media support several types of propagating modes, including longitudinal and transverse wave motion. Additionally, in elastic IWE the wave field is characterized at the free surface, as nondestructive access to the interior of the solid object is not straightforward. In this work, we provide a theoretical and numerical study showing that elastic IWE requires only knowledge of the traction incident on the faces of the target to make the physical boundaries transparent. We then present the proof of concept of elastic IWE in a one-dimensional (1D) beam. Section II begins by introducing the theory of elastic IWE. In Sec. III, we implement elastic IBCs in a numerical experiment to both verify the theory and aid in the understanding of its physical implementation in the laboratory. In Sec. IV, we propose a workflow which allows the first physical implementation of elastic IWE in 1D on a thin aluminum beam. 


\section{THEORY OF IMMERSIVE ELASTIC EXPERIMENTATION}

Immersing a physical experiment within a numerical experiment has two fundamental requirements. First, unwanted reflections (including mode conversions) from the boundaries of the physical experiment must be removed. Second, the wave field propagating in the physical domain must be allowed to interact with an arbitrary designed virtual domain.

In line with the first requirement, the work of Ting and Miksis [15] showed that nonreflecting boundary conditions can be found for a domain by recording the wave field on a closed surface and extrapolating it to the edges of the domain. Based on this idea, van Manen et al. [2] introduced a novel set of radiation boundary conditions which can be used to dynamically link a truncated modeling domain to a chosen, possibly much larger, nontruncated domain. All interactions of arbitrary scattering between the two domains are accounted for accurately. Such immersive boundary conditions (IBCs) are the core component to meeting both of the requirements for immersive experimentation [1].

We aim to link the virtual domain to the truncated physical domain by deriving an expression for the extended domain, i.e., the full domain incorporating both the virtual and physical domains, with a transparent boundary between the two. This is achieved by first finding an expression for the displacement in the extended domain $V_{\text {full }}$ in terms of an auxiliary state truncated by a free-surface boundary. Subsequently, we derive an expression for the laboratory state, defined as the domain $V_{\text {phy }}$. Together these two steps provide an expression for 3D elastic IBCs. Following the derivation of Broggini et al. [16] and Vasmel [17], we begin by defining two states of wave propagation, denoted $A$ and $B$, which are related via Betti's theorem with causality [18]:

$$
\begin{aligned}
\iiint_{V}\left[\mathbf{u}_{A}(\mathbf{x}, t) * \mathbf{f}_{B}(\mathbf{x}, t)-\mathbf{u}_{B}(\mathbf{x}, t) * \mathbf{f}_{A}(\mathbf{x}, t)\right] d V \\
=\oint_{S}\left[\mathbf{u}_{B}(\mathbf{x}, t) * \mathbf{T}\left(\mathbf{u}_{A}(\mathbf{x}, t), \mathbf{n}\right)\right. \\
\left.\quad-\mathbf{u}_{A}(\mathbf{x}, t) * \mathbf{T}\left(\mathbf{u}_{B}(\mathbf{x}, t), \mathbf{n}\right)\right] d S,
\end{aligned}
$$

where $*$ denotes temporal convolution. The body forces $\mathbf{f}_{A}$ together with the boundary conditions on the surface $S$ and initial conditions at time $t=0$ cause the displacement field $\mathbf{u}_{A}(\mathbf{x}, t)$. This displacement field is then related to the displacement field $\mathbf{u}_{B}(\mathbf{x}, t)$, caused by body forces $\mathbf{f}_{B}$ and generally different boundary conditions on $S$, as well as initial conditions at $t=0$. The terms $\mathbf{T}\left(\mathbf{u}_{A}, \mathbf{n}\right)$ and $\mathbf{T}\left(\mathbf{u}_{B}, \mathbf{n}\right)$ differentiate between the traction on surfaces normal to $\mathbf{n}$ due to the displacements $\mathbf{u}_{A}$ and $\mathbf{u}_{B}$ respectively.

The first step is to find an expression for the extended domain. Therefore, state $A$ is defined as the desired, unbounded state and can be seen as the domain $V_{\text {full }}$ in Fig. 1. In this state $S_{\text {rec/emt }}$ is transparent. State $B$ is defined as an auxiliary state truncated by a free-surface boundary. We let the source term $\mathbf{f}_{B}(\mathbf{x}, t)$ in state $B$ take the form of a body force $f_{\mathbf{B}}(\mathbf{x}, t)_{n}=$ $\delta(\mathbf{x}-\xi) \delta(t-\tau) \delta_{\text {in }}$, where $\delta(\mathbf{x}-\xi)$ is the Dirac delta function determining the spatial location, $\delta(t-\tau)$ determines the time, and $\delta_{\text {in }}$ the direction of the impulse [18]. This source term can be understood as the the unit impulse applied at

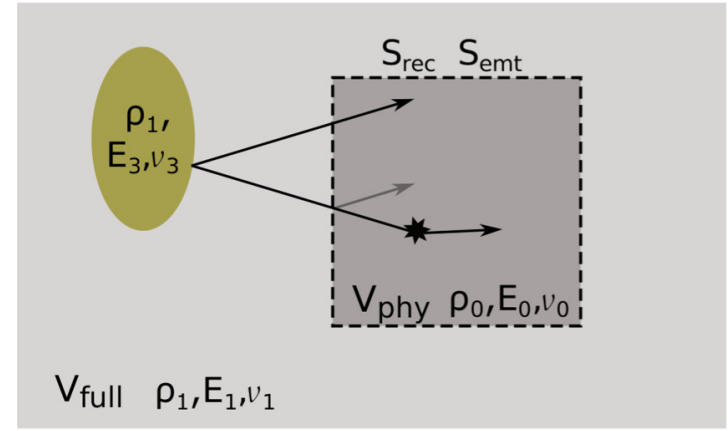

FIG. 1. Schematic of the two different domains referred to in IWE, defined by their respective elastic medium parameters: mass density $\rho$, Young's modulus $E$, and Poisson's ratio $v$. The physical domain $V_{\text {phy }}$ is bounded by a free surface (dashed black line). A source fired inside the physical domain causes waves to propagate towards and interact with the free-surface boundary, where they are recorded. Utilizing IBC theory, the physical free-surface boundary is made transparent and all free-surface reflections are removed (indicated by the light-gray arrow). The incident wave field is then propagated into the virtual domain where it interacts with any scatterers and is then introduced back into the physical medium. Both the recording $S_{\text {rec }}$ and emitting $S_{\text {emt }}$ surface are in line with the physical free surface of the target.

$\mathbf{x}=\xi$ at time $t=\tau$ in the $n$ direction and causes the elastodynamic Green's function displacement field $\mathbf{u}_{B}(\mathbf{x}, t)=$ $G_{\text {in }}(\mathbf{x}, t \mid \xi, \tau)$. We proceed by substituting these expressions into Eq. (1) and integrating with the delta function. By imposing a free-surface boundary condition on $S$ in state $B$, the traction term $\mathbf{T}\left(\mathbf{u}_{B}, \mathbf{n}\right)=c_{i j k l} n_{j} G_{k n, l}(\mathbf{x}, t \mid \xi, \tau)$ is zero. This leaves us with a way to express the displacement field $\mathbf{u}_{A}=$ $u_{\text {full }, n}(\mathbf{x}, t)$ inside the extended volume $V_{\text {full }}$ in terms of the Green's function of an auxiliary truncated state $\mathbf{u}_{B}(\mathbf{x}, t)=$ $G_{n i}^{\text {free }}(\mathbf{x}, t \mid \xi, 0)$, the source term $\mathbf{f}_{A}(\mathbf{x}, t)=f_{A, i}(\xi, t)$ in the extended domain, and the boundary traction $\mathbf{T}\left(\mathbf{u}_{A}, \mathbf{n}\right)=$ $\mathbf{T}\left(\mathbf{u}_{\text {full }}, \mathbf{n}\right)$ in the extended domain:

$$
\begin{aligned}
u_{\text {full }, n}(\mathbf{x}, t)= & \iiint_{V} f_{\text {full }, i}(\xi, t) * G_{n i}^{\mathrm{free}}(\mathbf{x}, t \mid \xi, 0) d V_{\xi} \\
& +\oint_{S} G_{n i}^{\mathrm{free}}(\mathbf{x}, t \mid \xi, 0) * T_{i}\left(\mathbf{u}_{\mathrm{full}}(\xi, t), \mathbf{n}\right) d S_{\xi} .
\end{aligned}
$$

To find an expression for the laboratory state $V_{\text {phy }}$ in Fig. 1, we truncate domain $A$ by a free-surface boundary along $S_{\text {rec/emt }}$. This boundary is in line with the edges of our experimental target in the laboratory. The characteristics of auxiliary state $B$ stay the same as before. However, also truncating the domain in state $A$ by a free surface causes the traction term $T_{i}\left(\mathbf{u}_{A}(\xi, \tau), \mathbf{n}\right)$, with $\mathbf{n}$ normal to the boundary $S_{\text {rec/emt }}$, to also be zero. Consequently, the second representation is

$$
u_{\mathrm{phy}, n}(\mathbf{x}, t)=\iiint_{V} f_{\mathrm{phy}, i}(\xi, t) * G_{n i}^{\mathrm{free}}(\mathbf{x}, t \mid \xi, 0) d V_{\xi} .
$$

Comparing Eqs. (2) and (3), we find that the surface integral term has vanished in Eq. (3). Furthermore, if the same source is excited in both domains $V_{\text {full }}$ and $V_{\text {phy }}$, we find that the right-hand side of (3) is equal to the first integral on the 
right-hand side of Eq. (2), which leads to

$$
\begin{aligned}
u_{\text {full }, n}(\mathbf{x}, t)= & u_{\text {phy }, n}(\mathbf{x}, t) \\
& +\oint_{S} G_{n i}^{\text {free }}(\mathbf{x}, t \mid \xi, 0) * T_{i}\left(\mathbf{u}_{\text {full }}(\xi, t), \mathbf{n}\right) d S_{\xi} .
\end{aligned}
$$

Hence, the difference between the displacement in the extended domain $u_{\mathrm{full}, n}(\mathbf{x}, t)$ and the truncated, physical domain $u_{\mathrm{phy}, n}(\mathbf{x}, t)$ is equal to the integral over the bounding surface $S_{\text {rec/emt }}$ of the temporal convolution between the Green's function of the truncated domain and the normal component of the traction measured on the surface $S_{\text {rec/emt }}$. It follows that the displacement in the desired (unbounded) state can be made equal to the displacement in the truncated domain, i.e., the physical target in the laboratory, by adding the surface integral term from Eq. (4):

$$
u_{n}^{\mathrm{IBC}}(\mathbf{x}, t)=\oint_{S} G_{n i}^{\mathrm{free}}(\mathbf{x}, t \mid \xi, 0) * T_{i}\left(\mathbf{u}_{\mathrm{full}}(\xi, t), \mathbf{n}\right) d S_{\xi} .
$$

Equation (5) can be interpreted as an expression for the wave field in the $n$ direction at an arbitrary point in the interior, generated by point force sources on $S$, weighted by the traction term $T_{i}\left(\mathbf{u}_{\text {full }}, \mathbf{n}\right)$. Thus the wave field is the result of applying directed force sources along the boundary $S_{\text {emt }}$ with signatures equal the normal traction of the wave field arriving at $S$ from both the physical and virtual domains. Hence, the traction $T_{i}\left(\mathbf{u}_{\text {full }}, \mathbf{n}\right)$ linking the two domains comprises both $T_{i}^{\text {inc }}\left(\mathbf{u}^{\text {inc }}, \mathbf{n}\right)$, the traction corresponding to the wave field incident on the free surface in the physical domain, and $T_{i}^{\text {virt }}\left(\mathbf{u}^{\text {inc }}, \mathbf{n}\right)$, the traction corresponding to interactions of the wave field with the virtual domain.

Applying $T_{i}^{\text {inc }}\left(\mathbf{u}^{\text {inc }}, \mathbf{n}\right)$ as a boundary condition on the free surface of the target cancels any reflections and modeconverted energy at the boundary. To determine the traction corresponding to the wave field incident on the free surface in the physical domain $T_{i}^{\text {inc }}\left(\mathbf{u}^{\text {inc }}, \mathbf{n}\right)$, the full three-component wave field associated with a source inside the elastic object is recorded as a function of time at the free surface along $S_{\text {rec }}$ (see Fig. 1). The recording $\mathbf{u}_{\text {tot }}(\mathbf{x}, t)$ is a superposition of the incident $\mathbf{u}_{\mathrm{inc}}(\mathbf{x}, t)$ and reflected $\mathbf{u}_{\mathrm{sc}}(\mathbf{x}, t)$ wave fields at the free surface: $\mathbf{u}_{\mathrm{tot}}(\mathbf{x}, t)=\mathbf{u}_{\mathrm{inc}}(\mathbf{x}, t)+\mathbf{u}_{\mathrm{sc}}(\mathbf{x}, t)$. The total wave field must be separated into its respective incident and reflected components. One promising method of wave field separation is proposed by Thomsen et al. [19]. Using the wave field incident on the free surface $\mathbf{u}_{\text {inc }}(\mathbf{x}, t)$, the associated traction is calculated via the elastic constitutive relations: $T_{i}^{\text {inc }}\left(\mathbf{u}^{\text {inc }}, \mathbf{n}\right)=c_{i j k l} \partial_{k} u_{l}^{\text {inc }} n_{j}$. Hence, the medium properties $c_{i j k l}$ at the recording boundary must be known. Note that it is not necessary to separate the wave field into its longitudinal and transverse modes.

The second constituent $T_{i}^{\mathrm{virt}}\left(\mathbf{u}^{\mathrm{inc}}, \mathbf{n}\right)$ is computed by discretized convolutions of the incident wave field $\mathbf{u}_{\text {inc }}(\mathbf{x}, t)$ with Green's functions characterizing the numerical medium [1,2]. Together, the time series of both traction terms are applied as signatures of force point sources at the boundary $S_{\text {emt }}$, thereby removing any reflections or scattering at the boundary and incorporating propagation between the two domains. Thus, linking physical and numerical experiments is possible by continuously recording and emitting signals at the free surface of the target. Unlike the acoustic implementation of IWE [3], the recording $S_{\text {rec }}$ and emitting $S_{\text {emt }}$ surfaces overlap in the elastic case. For this reason, the wave field that needs to be applied on the boundary of the target cannot be predicted in real time. Hence, the IBCs must be designed recursively through a sequence of experiments until the desired experimental state is reached. Finally, note that Eq. (5) requires knowledge of only the wave field incident on the boundary $S_{\text {rec }}$ and its interactions with the virtual domain. Thus, the physics of wave propagation in the physical experiment can be different from that in the numerical simulation, and can even be unknown.

\section{NUMERICAL IMPLEMENTATION}

First, we numerically verify the implementation of elastic immersive experimentation. Using the finite element based structural mechanics module in COMSOL MULTIPHYSICS ${ }^{\circledR}$ [20], we simulate mechanical wave propagation in a thin aluminum beam model. The model provides good experimental control, by ensuring propagation of uncoupled longitudinal and flexural modes within the beam and well-separated arrivals. The beam is set to a length of $x=200 \mathrm{~cm}$, height $y=3 \mathrm{~cm}$, and width $z=3 \mathrm{~cm}$. Furthermore, the model has a density $\rho=$ $2700 \mathrm{~kg} / \mathrm{m}^{3}$, Young's modulus $E=70 \mathrm{GPa}$, and Poisson's ratio $v=0.33$. The side of the beam where the wave field is excited $(x=0 \mathrm{~cm})$ is referred to as the SRC side. The opposite side $(x=200 \mathrm{~cm})$, where we aim to remove reflections, is referred to as the IBC side. We define a small, $1-\mathrm{cm}$-thin region at the SRC side of the beam where we apply a body load [see Fig. 2(a)]. The force per unit volume $\left(\mathrm{N} / \mathrm{m}^{3}\right)$ uses a Ricker source-time function of central frequency $f_{c}=10 \mathrm{kHz}$ and a maximum amplitude of $0.1 \mathrm{~N} / \mathrm{m}^{3}$. It acts only on the $x$ axis of the beam. This ensures that a purely compressional wave propagates along the $x$ direction of the beam. The simulation is run using a time dependent solver. Numerical stability is ensured by a time step of $d t=1 /\left(20 f_{c}\right)=5 \times 10^{-6} \mathrm{~s}$ and maximum element size of the tetrahedral mesh of $\lambda / 6$, with $\lambda=\sqrt{E / \rho} / f_{c}$.

The dark red line in Fig. 2(b) shows the resulting displacement $u_{x}$, evaluated exactly in the middle of the beam at $1 \mathrm{~m}$ distance from the emitting boundary, as indicated by a black triangle in Fig. 2(a). One can clearly distinguish the first arrival, which is the incident wave, and the second arrival, which in turn is the wave reflected at the opposite end of the beam. We also evaluate the normal traction $T_{x}$ (light blue line) acting on the cross section in the middle of the beam. According to Eq. (5), we must apply the incident traction acting on the IBC side as a body force to cancel the reflected wave field. To circumvent the necessity of separating the traction measured at the end of the beam into its incident and reflected components, we extend the beam by $1 \mathrm{~m}$ and extract just the traction incident at $2 \mathrm{~m}$ from the simulation. This traction, scaled by the volume of the $1-\mathrm{cm}$-thin region at the IBC end of the beam, is then injected as the IBC body force in the original model of $2 \mathrm{~m}$ length. Figure 2(c) shows the resulting displacement and traction at $1 \mathrm{~m}$. We observe that applying the incident traction at the IBC side causes a longitudinal wave to propagate within the beam with flipped polarity when compared to the reflected wave in Fig. 2(b). 


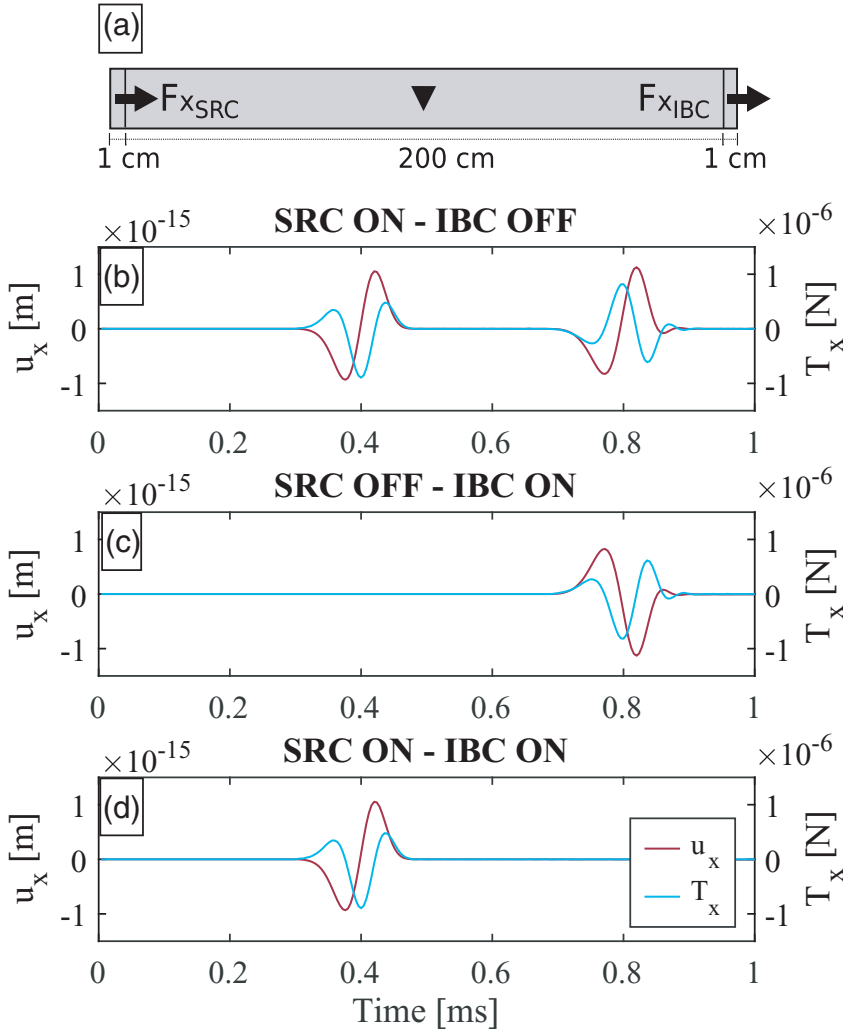

FIG. 2. (a) 1D schematic of the 200-cm-long 3D comsol model. The body load force $F x_{\mathrm{SRC}}$ excites a longitudinal wave within the beam. The propagating wave field is evaluated exactly in the middle of the beam, as indicated by the black triangle. The body load force $F x_{\mathrm{IBC}}$ applies the immersive boundary condition on the opposite side of the beam. We evaluate both the displacement in $x$ direction $u_{x}$ (dark red line) and the resulting normal traction $T_{x}$ (light blue line) at $100 \mathrm{~cm}$ when (b) only the SRC side is active, (c) only the IBC side is active, and (d) both the SRC and IBC are active and the reflected wave is canceled.

Finally, we apply both the SRC and IBC terms at the same time. As a result, the reflected wave is canceled and only the incident wave propagates within the aluminum beam [see Fig. 2(d)]. Hence, we are able to verify general elastic IBC theory via the example of a 1D beam. Specifically, confirming that introducing a body force at the free-surface boundary of an elastic target with the signature of the incident traction measured at the boundary ensures removal of any free-surface reflections.

\section{EXPERIMENTAL IMPLEMENTATION}

For the experimental demonstration of elastic immersive wave experimentation, we consider an aluminium beam with same dimensions as in the numerical implementation. Again this ensures uncoupled propagation of longitudinal and flexural components and allows us to investigate their excitation and cancellation individually, as well as the combination of the two. Both the SRC and IBC sources are multiaxis PICA 153.05 shear actuators [21]. Using these piezoelectric actuators, we can excite motion in either the $x, y$, or $z$ direction of the beam or a combination of the three. The actuators are

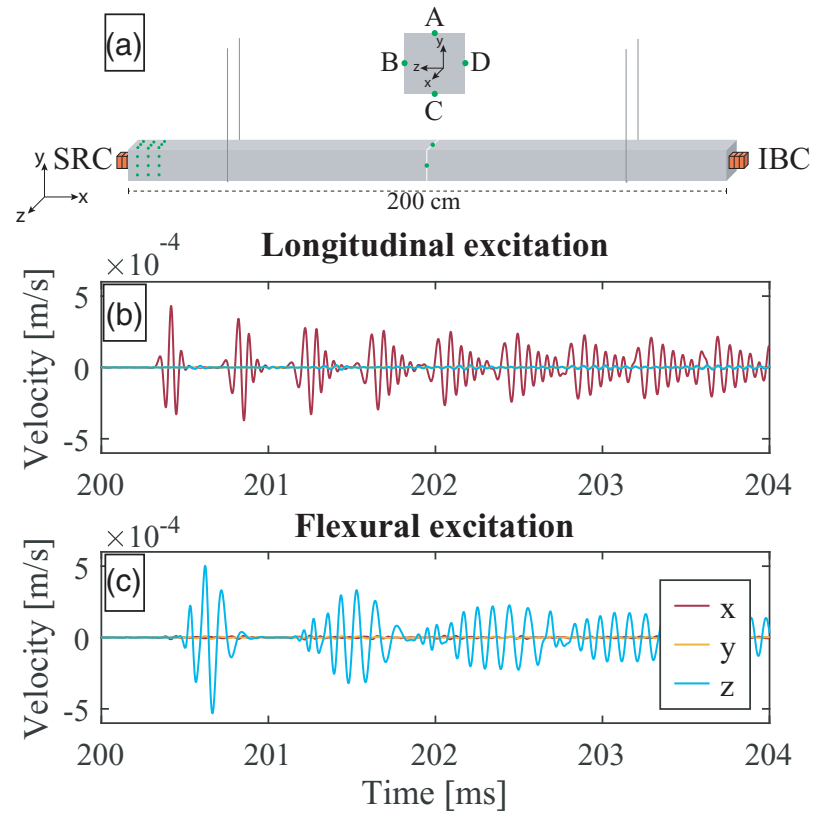

FIG. 3. (a) Experimental setup: The 200-cm-long and 3-cm-thick aluminum beam is supported by two thin ropes. A three-component piezoelectric actuator is attached to either end of the beam. To visualize the wave field propagating along the entire beam (see Supplemental Material movies [25], Figs. 5 and 7) the LDV measures the particle velocity along a dense grid of scan points $1 \mathrm{~cm}$ apart on two faces of the beam as indicated by the green dots at the left end of the schematic. The measurements made to determine the IBCs are acquired at and around $100 \mathrm{~cm}$ with the cross section showing the four scan points A-D located in the middle of the individual faces of the aluminum beam. The particle velocity at points A-D is measured at $100 \mathrm{~cm}$ and the average of the four points is displayed when (b) exciting a longitudinal wave and (c) exciting a flexural wave.

driven by a field-programmable gate array (FPGA) analog output $(\mathrm{A} / \mathrm{O})$ system and the injected signal is amplified using a PiezoDrive PD32 amplifier [22]. We measure the wave field along the beams surface using a Polytec PSV-500-3D scanning vibrometer [23]. The laser Doppler vibrometer (LDV) allows us to measure the $x, y$, and $z$ components of particle velocity of the propagating wave field. To improve the signalto-noise ratio of the measurements, we add reflective tape along the beam and each point is scanned 100 times to average the measurements. The beam is held up horizontally by two thin ropes $30 \mathrm{~cm}$ from the edges which do not introduce any noticeable reflections or mode conversions. Figure 3(a) depicts a schematic of the described experimental setup, also indicating the location of the scan points via the green points labeled A-D at $100 \mathrm{~cm}$. Figures 3(b) and 3(c) display the 0.1 to $50 \mathrm{kHz}$ bandpass filtered time series of the overall movement of the cross section, determined by averaging all four measurements. Figure 3(b) shows how exciting the SRC actuator along the $x$ axis causes only longitudinal waves to propagate along the $x$ direction of the beam. Figure 3(c), however, displays a time series with flexural motion only along the $z$ axis, caused by excitation of the $z$ component of the SRC. 
To achieve our objective of canceling reflections of both longitudinal and flexural wave fields at one end of the aluminum beam, we must undergo several steps. First, the recorded wave field is separated into its left- and rightward propagating components. We bypass the necessity to separate the superposition of the incident and reflected waves measured directly at the ends of the beam by utilizing the wave field separation method proposed by Sønneland et al. [24]. The method is based on using a vertical receiver configuration in seismic exploration and assumes that at each scan point along the beam the wave field can be expressed as the sum of rightward and leftward propagating components. When exciting the beam on the left side via the SRC, the incident wave field travels to the right and the scattered to the left. We measure the wave field propagating along the beam at two positions, $x_{1}$ and $x_{2}$, to apply the wave field separation algorithm described in Appendix A. When exciting longitudinal and flexural components simultaneously the propagating flexural wave causes additional arrivals to be present in the $x$ component measured. We average four measurements obtained on opposite sides of the beam, i.e., a measurement at $x=100 \mathrm{~cm}$ at points A, B, C, and D [see Fig. 3(a)]. As a result, any arrivals caused by the flexural movement of the beam are canceled out of the $x$ component, leaving only longitudinal motion. Additionally, any longitudinal motion is canceled out of the flexural movement, i.e., the $z$ component. The resulting time series can be seen as the superposition of individually exciting either longitudinal or flexural waves [see Fig. 4(a)]. Different distances $\Delta x=x_{2}-x_{1}$ are required to accurately separate either the longitudinal or flexural waves due to their distinct dominant wavelengths. The longitudinal wave field is separated using measurements at $x_{1}=95 \mathrm{~cm}$ and $x_{2}=$ $100 \mathrm{~cm}(\Delta x=5 \mathrm{~cm})$. The flexural wave field on the other hand, is separated using measurements at $x_{1}=99 \mathrm{~cm}$ and $x_{2}=100 \mathrm{~cm}(\Delta x=1 \mathrm{~cm})$. Figure 4(b) shows a time series of the resulting rightward and Fig. 4(c) the leftward propagating wave fields at $100 \mathrm{~cm}$ resulting from a simultaneous excitation of longitudinal and flexural waves. The respective individual incident and reflected arrivals measured in the middle of the beam can then be back or forward propagated to either end of the beam using the experimentally determined longitudinal and flexural wave numbers (see Appendix B).

Both the theory derived in Sec. II and the numerical modeling results in Sec. III show that we need to apply the incident traction at the IBC end of the beam. However, this assumes access to an idealized point force source. In the laboratory, we are limited to the three-component piezoelectric actuator, which applies a boundary displacement proportional to the input voltage. Hence, we need to determine a transfer function between the the piezoelectric actuator and a point force source. However, in the numerical implementation in Sec. III, we observe that applying the incident traction at the IBC side causes a wave with polarity opposite to that of the reflected wave to propagate within the beam [see Fig. 1(c)]. Thus, instead of designing a piezoelectric actuator acting as a ideal point source to apply the incident traction, we construct the displacement $u^{\mathrm{IBC}}$, which needs to be applied by the IBC actuator to cancel the reflection at the boundary. Appendix C explains how $u^{\mathrm{IBC}}$ is constructed. It is not necessary to rely on this method when constructing the IBCs in $2 \mathrm{D}$ or $3 \mathrm{D}$, as
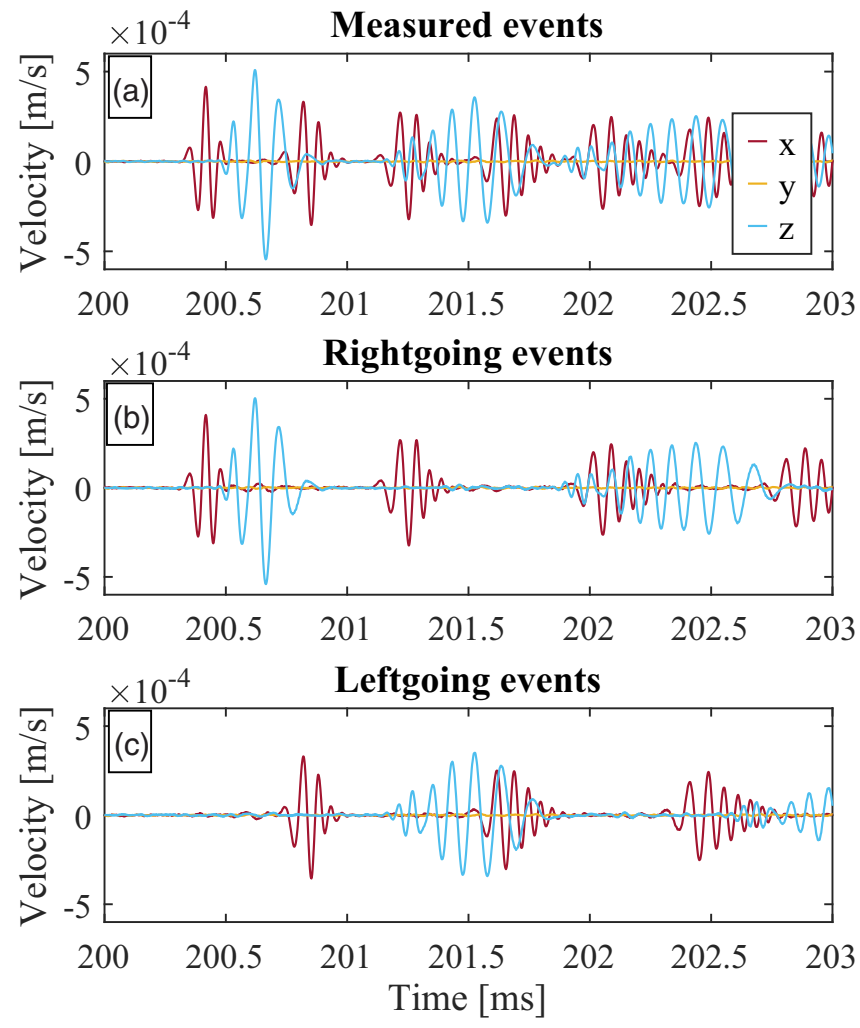

FIG. 4. Longitudinal and flexural waves are excited simultaneously at the SRC side of the aluminum beam. The propagating wave field is measured at $100 \mathrm{~cm}$ on sides A, B, C, and D of the beam (see Fig. 3). (a) The average propagation of the cross section, leaving us with purely longitudinal movement in the $x$ component and flexural movement in the $z$ component. Panels (b) and (c) depict the right- and leftgoing propagating arrivals after successful wave field separation.

knowledge of the incident traction provides the correct vector of signatures to cancel the different incident wave types or different types of mode-converted reflections simultaneously.

\section{A. Canceling the reflection at one side of the aluminum beam}

In the following, we cancel the reflections at the IBC boundary when exciting either longitudinal or flexural waves individually and then while simultaneously exciting both wave propagation modes. In each case, we apply a Ricker source wavelet of $10 \mathrm{kHz}$ central frequency to the SRC. We then determine the canceling wave field $u^{\mathrm{IBC}}$ and use the LDV system to scan two full sides of the beam, once with just the source active (SRC ON, IBC OFF) and once with the both the source and the IBC wave field active (SRC ON, IBC ON).

First, we excite a longitudinal wave mode. Figure 5 visualizes the recorded in-plane $x$ component measured by the LDV along the middle of side B [see Fig. 3(a)], with individual scan points spaced $1 \mathrm{~cm}$ apart. In Fig. 5(a) one can observe how the wave propagates between the SRC end on the left side of the beam and the IBC end on the right. Once the IBC wave field is introduced, the reflections at the right boundary cease to exist, as can be seen in Fig. 5(b), and we observe only one wave field propagating from the left to the right. The time series of a single scan point at $x=100 \mathrm{~cm}$ shows how 

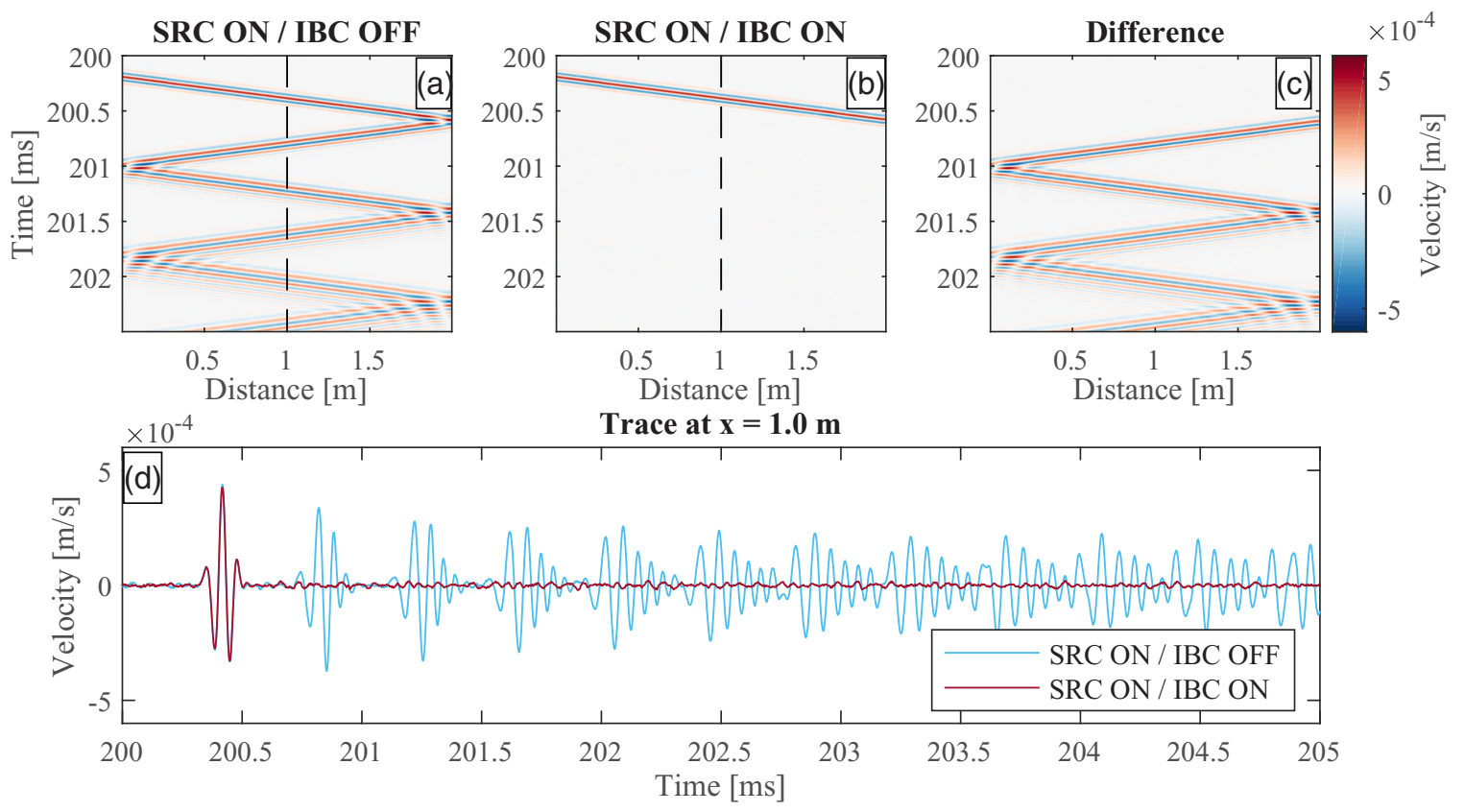

FIG. 5. A longitudinal wave mode is excited along the $x$ component of propagation at the SRC side (also see Supplemental Material Movie 0 [25]). The reflected wave field is effectively canceled via injection of a canceling wave field at the IBC side. The wave field is measured from 0 to $200 \mathrm{~cm}$ along the middle of one side of the beam and displayed as a time-distance panel: (a) Truncated time-distance panel of $x$-component time series. The wave propagates back and forth between the ends of the beam when the IBC is turned OFF; (b) the reflected wave field is canceled when the IBC is turned ON; (c) difference between the IBC being OFF and ON. (d) A single time series of the $x$ component of the wave field at $x=100 \mathrm{~cm}$ when the IBC is OFF vs ON.

only the first arrival is present in the recorded data [Fig. 5(d)]. The same observations can be made when exciting a purely flexural wave field along the $z$ direction at the SRC side and applying the respective canceling wave field at the IBC side of the beam [Figs. 6(a)-6(d)]. Compared to the longitudinal case, we observe more residual energy when canceling the reflected flexural wave. We attribute this difference to the flexural wave mode being more dispersive, as can be observed in Fig. 6. Therefore, designing the source transfer function to remove the impulse response of the IBC actuator (see Appendix C) is more complex, resulting in an imperfect match of the wave field injected by the IBC actuator to the desired $u^{\mathrm{IBC}}$ wave field. Finally, we excite both longitudinal and flexural wave modes simultaneously. The immersive boundary conditions are determined individually for each excitation direction but applied simultaneously to the respective component of the piezoelectric actuator on the IBC side. Figure 7 quantitatively shows that reflections of both wave modes are effectively canceled by displaying a time series of the $x, y$, and $z$ components of the particle velocity at $100 \mathrm{~cm}$. In all three cases the IBC implementation proves to be effective in removing broadband boundary reflections.

\section{B. Introducing a virtually scattered wave field}

Finally, we demonstrate the ability of virtually extending the dimensions of the physical aluminum beam by injecting into the physical domain a wave field scattered in a virtual environment. Figure 8(a) shows a schematic of the desired result. We excite a longitudinal wave at the source side of the aluminum beam. As previously described, we design an
IBC wave field to cancel the reflection at the right side of the aluminum beam. Additionally, we window the wave field incident at the middle of the beam and forward propagate it to the IBC boundary. From here, we forward propagate the wave field $75 \mathrm{~cm}$ into a virtual domain, convolve it with the theoretical reflection coefficient of a free surface boundary $(R=1)$ and forward propagate the reflection an additional $75 \mathrm{~cm}$ back to the position of the physical IBC boundary. At this point the wave field is injected back into the physical domain. The injected wave field then propagates towards and reflects off the SRC boundary on the left side of the beam. The interaction with the physical boundary causes a deformation of the wave form. Hence, we must repeat the procedure to once again cancel the reflection and propagate the wave field into the virtual domain. Thus, we design a second set of wave forms which both cancel the reflected wave and interact with the virtual domain. Consequently, the immersive boundary conditions are designed iteratively.

The naturally occurring arrivals are depicted in Fig. 8(b), whereas Fig. 8(c) shows the waves propagating along the beam when virtually extending the physical medium on the right side. The previously reflected arrivals are gone and new, delayed arrivals are introduced. For a quantitative comparison between both configurations, Fig. 8(d) displays a time series of wave fields (a) and (b) at $100 \mathrm{~cm}$. We observe that the naturally occurring arrivals are almost perfectly canceled.

\section{Considerations for extension to 3D IWE}

The realization of IWE in 3D will bring additional challenges. First, the IBCs will require a dense coverage of 

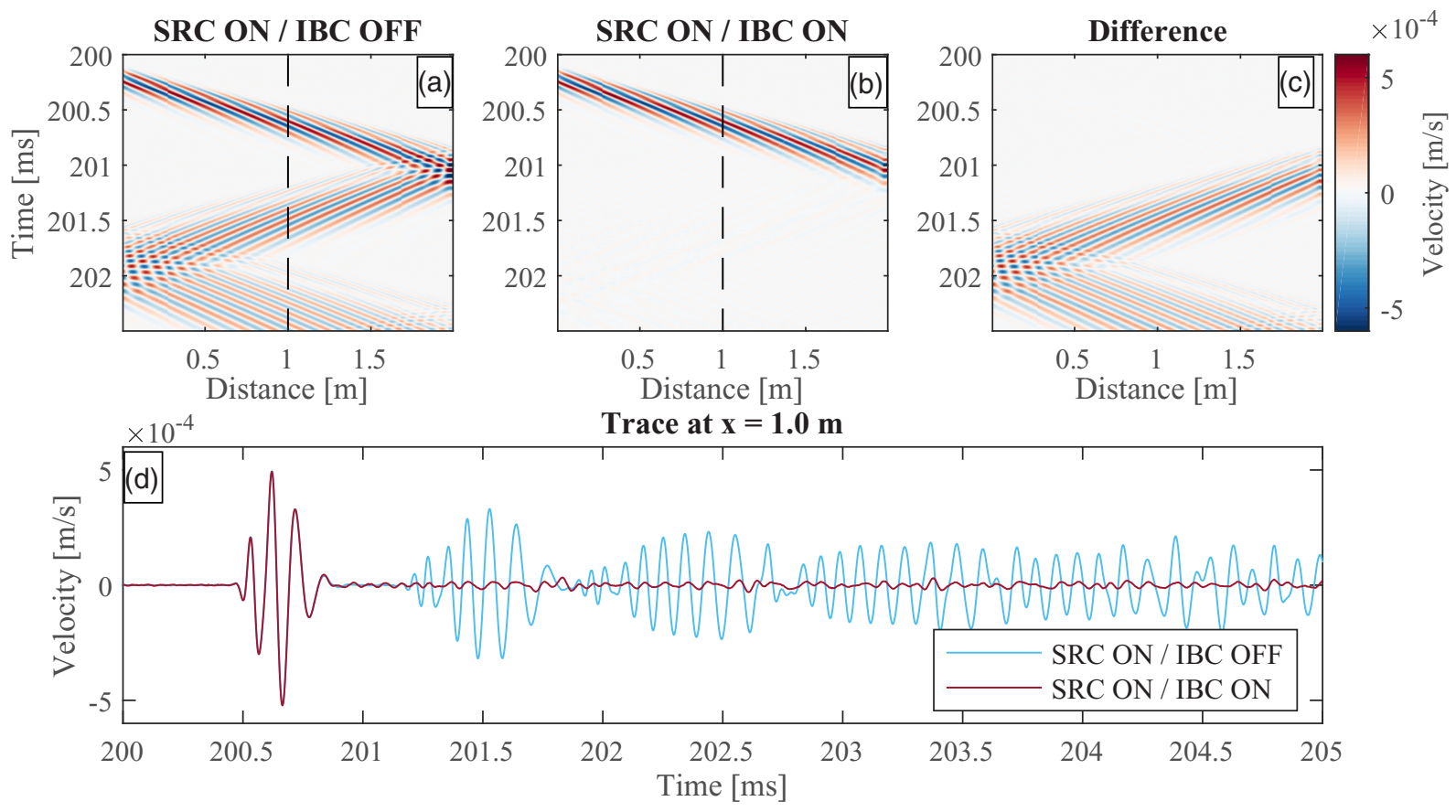

FIG. 6. A flexural wave mode is excited along the $z$-component of propagation at the SRC side (also see Supplemental Material Movie 1 [25]). The reflected wave field is effectively canceled via injection of a canceling wave field at the IBC side. The wave field is measured from 0 to $200 \mathrm{~cm}$ along the middle of one side of the beam and displayed as a time-distance panel: (a) Truncated time-distance panel of $z$-component time series. The wave propagates back and forth between the ends of the beam when the IBC is turned OFF; (b) the reflected wave field is canceled when the IBC is turned ON; (c) difference between the IBC being OFF and ON. (d) A single time series of the $z$ component of the wave field at $x=100 \mathrm{~cm}$ when the IBC is OFF vs ON.

three-component piezoelectric actuators on the target faces distributed according to Nyquist's sampling criterion [26]. Therefore, it will be necessary to fully understand the foot-
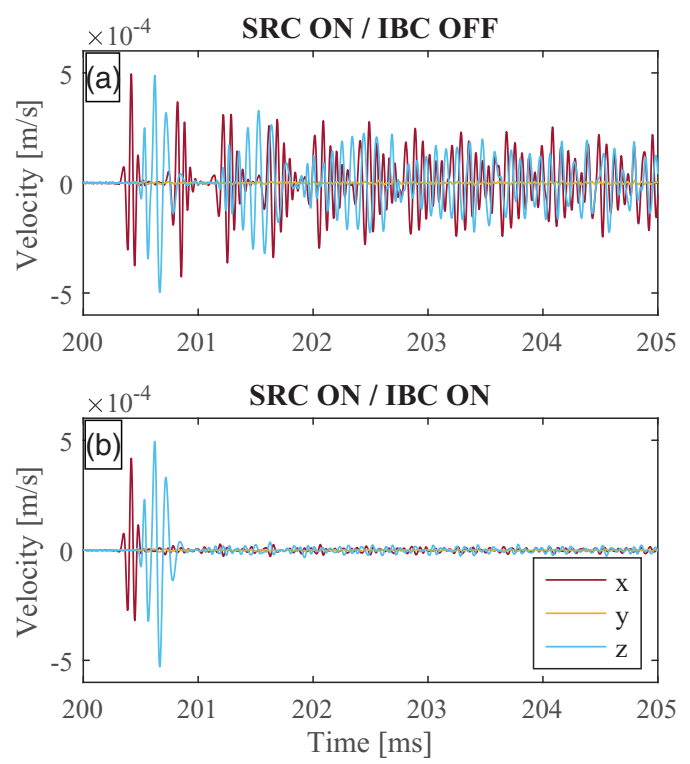

FIG. 7. Longitudinal and flexural wave modes are excited simultaneously along the $x$ and $z$ components of propagation at the SRC side (also see Supplemental Material Movie 2 [25]). The wave field is measured at $x=100 \mathrm{~cm}$ with (a) the IBC turned OFF and (b) the IBC turned ON leading to an effective cancellation of the reflected wave field. print of the actuators on the wave field propagating at the surface of the object, i.e., their effect on the boundary condition and scattering, which can be studied using an LDV system for instance. Furthermore, it will be necessary to characterize the radiation pattern of the actuators and possibly compensate for any directivity present [27]. The placement of the transducers will need to be staggered with respect to the LDV recordings. Hence, the wave field must be reconstructed at the point of injection by interpolating the LDV point measurements $[28,29]$. In the $1 \mathrm{D}$ experimental demonstration of IWE presented, we use the LDV to measure the wave propagation along the beam. This enables us to circumvent the necessity to separate the superimposed incident and reflected wave fields at the ends of the beam by determining the rightand leftward propagating events along the beam. However, it requires that we determine the experimental wave number to accurately redatum those wave field components to the ends of the beam. When extending IWE to 3D the wave field needs to be separated into its incident and reflected components using only recordings of the velocity at the freesurface faces of the target. We plan to utilize the wave field injection based approach introduced by Thomsen et al. [19], which only relies on velocity recordings made at the free surface and can be applied to target geometries with sharp corners, such as a rock cube. Once the full three-component incident wave field is characterized, its horizontal and vertical derivatives can be used to compute the time series of the incident traction vector to be applied to the faces of the target [30]. Subsequently, a transfer function should be established such that the piezoelectric actuators behave as body force 

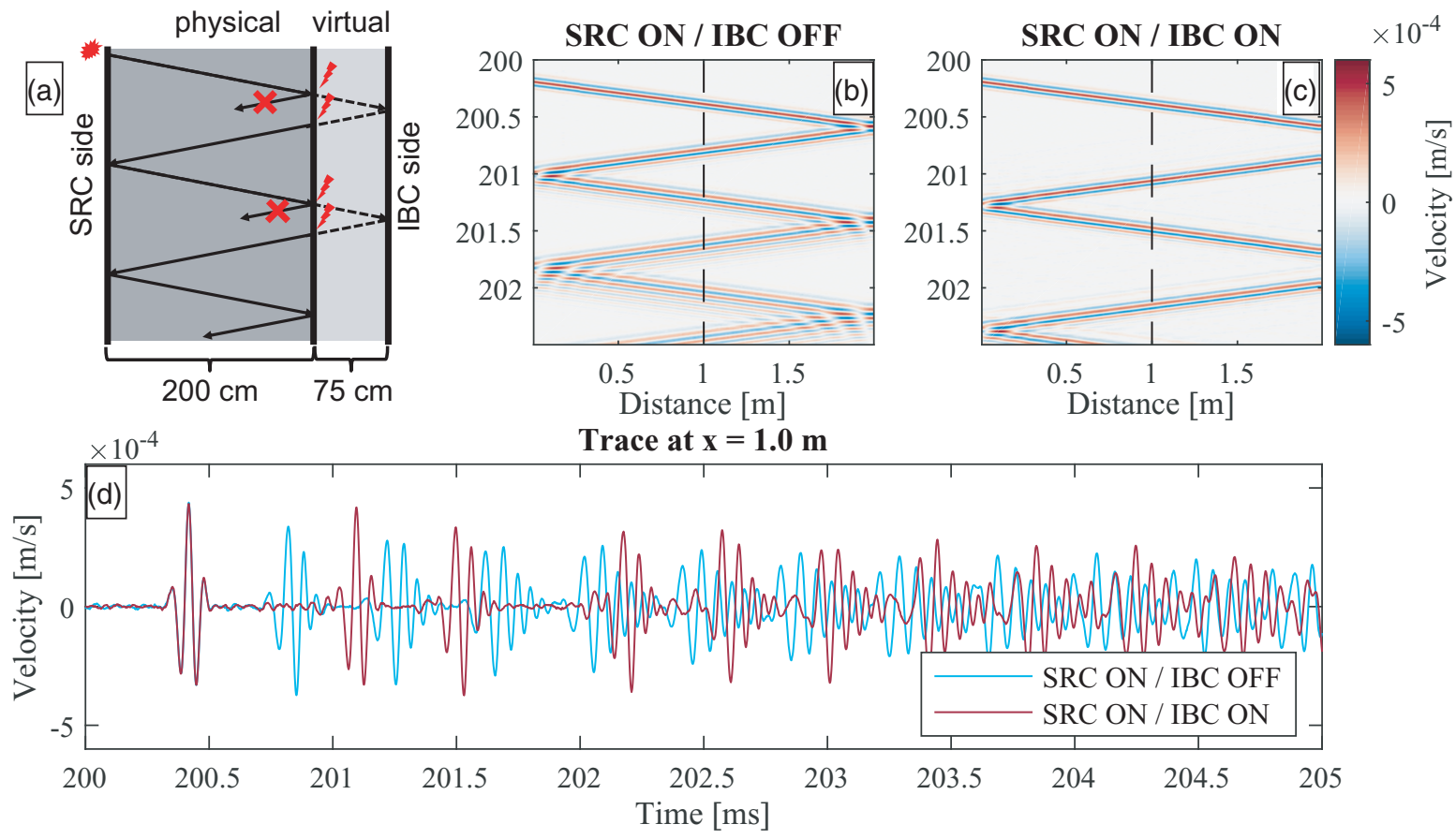

FIG. 8. (a) Schematic of the steps taken to virtually extend the physical medium by $75 \mathrm{~cm}$ through canceling the first two reflections at the IBC boundary and introducing new reflected arrivals from the virtual domain. A longitudinal wave mode is excited along the $x$-component of propagation at the SRC side (also see Supplemental Material Movie 3 [25]). The wave field is measured from 0 to $200 \mathrm{~cm}$ along the middle of one side of the beam: (b) Time-distance panel of $x$-component time-series. The wave reflects between the ends of the beam when the IBC is turned OFF. (c) The first two reflected events are canceled and virtual reflections are introduced when the IBC is turned ON. (d) Single time-series of the $x$-component of the wave field at $\mathrm{x}=1.0 \mathrm{~m}$ when the IBC is OFF vs. ON.

sources. The immersive boundary conditions must then be set up iteratively, while always accounting for new interactions of waves within and between the physical and numerical domains, thereby leading to a complete elastic immersive experiment.

\section{CONCLUSION}

We have presented an immersive wave propagation experiment in an elastic medium. Through a theoretical derivation of elastic immersive boundary conditions and a numerical verification, we showed that by characterizing the incident wave field at the free surface of the solid target, reflections at the boundary can be canceled and interactions of the wave field between the physical and a virtual domain can be fully accounted for. In a proof of concept, we actively cancel waves reflected at the free surface end of a thin aluminum beam for individually excited broadband longitudinal and flexural wave modes, as well as the simultaneous excitation of the two. Furthermore, we virtually extend the physical aluminum beam, by making the physical free-surface boundary transparent to the propagating wave and introducing interactions between the physical domain and a chosen virtual domain.

The ability to make the physical boundaries transparent to propagating waves via immersive wave experimentation removes the wavelength limit in elastic laboratory experimentation, thus allowing the study of complex wave fields with wavelengths which can even be larger than the target.
Additionally, immersive wave experimentation provides full experimental control so that, for instance, waves with more than one interaction with the free surface can be switched on or off in the laboratory. This is of particular interest in the development of new focusing and imaging techniques, with applications in seismology and potentially also in areas such as medical imaging and material testing.

\section{ACKNOWLEDGMENTS}

The authors would like to thank two anonymous reviewers for their valuable feedback. Furthermore, the first author thanks Erik Koene, Theodor Becker, and Xun Li for insightful discussions and suggestions. This research was supported by the Horizon 2020 ERC Grant No. 694407-MATRIX.

\section{APPENDIX A: SEPARATING THE INCIDENT AND SCATTERED WAVE FIELDS MEASURED ALONG THE BEAM}

Following Sønneland et al. [24], at each scan point along the beam the wave field $s(x, t)$ can be expressed as the sum of leftward $l(x, t)$ and rightward $r(x, t)$ propagating components. Fourier transforming gives

$$
S\left(x_{i}, \omega\right)=L\left(x_{i}, \omega\right)+R\left(x_{i}, \omega\right) .
$$

For convenience, the angular frequency term, $\omega$, is henceforth omitted. To propagate the $L\left(x_{1}\right)$ and $R\left(x_{1}\right)$ components 
from $x_{1}$ to $x_{2}$ the following one-way wave extrapolators are used:

$$
\begin{aligned}
& L\left(x_{1}\right)=W_{L}\left(x_{1}, x_{2}\right) L\left(x_{2}\right), \\
& R\left(x_{1}\right)=W_{R}\left(x_{1}, x_{2}\right) R\left(x_{2}\right),
\end{aligned}
$$

with

$$
\begin{gathered}
W_{L}\left(x_{1}, x_{2}\right)=\exp \left(-i k_{\mathrm{L}, \mathrm{F}} \Delta x\right), \\
W_{R}\left(x_{1}, x_{2}\right)=\exp \left(i k_{\mathrm{L}, \mathrm{F}} \Delta x\right) .
\end{gathered}
$$

Inserting Eqs. (A2a) and (A2b) into Eq. (A1) leads to the following two equations, which can be used to find the leftand rightgoing components of the wave field at $x_{2}$ :

$$
\begin{aligned}
& L\left(x_{2}\right)=\frac{W_{R}\left(x_{1}, x_{2}\right) S\left(x_{2}\right)-S\left(x_{1}\right)}{W_{R}\left(x_{1}, x_{2}\right)-W_{L}\left(x_{1}, x_{2}\right)}, \\
& R\left(x_{2}\right)=\frac{S\left(x_{1}\right)-W_{L}\left(x_{1}, x_{2}\right) S\left(x_{2}\right)}{W_{R}\left(x_{1}, x_{2}\right)-W_{L}\left(x_{1}, x_{2}\right)} .
\end{aligned}
$$

Using these one-way wave extrapolators the measured wave field at $x_{2}$ is both forward and backward propagated to position $x_{1}$. Subtracting the redatumed wave field from the one measured at $x_{1}$ provides us with either the rightgoing or leftgoing components of the wave field. This method of wave field separation assumes no mode conversions of the wave field between the two measuring positions [24].

\section{APPENDIX B: DETERMINING THE EXPERIMENTAL WAVE NUMBER}

Our proposed workflow requires us to accurately redatum, i.e., forward and back propagate, any arrivals measured along the beam to either end of the beam. This is achieved by considering plane wave expressions for the longitudinal and flexural components:

$$
U(x, \omega)=U_{0} \exp \left(i k_{\mathrm{L}, \mathrm{F}} x\right),
$$

in which $k_{\mathrm{L}}$ and $k_{\mathrm{F}}$ describe the longitudinal and flexural wave numbers. To experimentally determine $k_{\mathrm{L}}$ and $k_{\mathrm{F}}$, we excite either a longitudinal or flexural wave with the signature of a Ricker wavelet of central frequency $10 \mathrm{kHz}$ at the SRC side of the aluminum beam and measure the propagating wave field at 50 and $100 \mathrm{~cm}$. For both measurements, we window the first arriving wave form and compute its Fourier transform, giving us $U_{50}$ and $U_{100}$.

We consider nondispersive propagation of longitudinal waves. Therefore, the longitudinal wave number is $k_{\mathrm{L}}=$ $\omega / c_{\mathrm{Lt}}$, were $\omega=2 \pi f$ is the angular frequency and $c_{\mathrm{Lt}}$ the theoretical longitudinal wave speed. To determine $c_{\mathrm{Lt}}=\sqrt{E_{\mathrm{t}} / \rho}$, we begin with an initial guess of the Young's modulus, $E_{\mathrm{t}}=70 \mathrm{GPa}$. The density is calculated from the volume and weight of the beam as $\rho=2722 \mathrm{~kg} / \mathrm{m}^{3}$. We then compute the transfer function between $U_{50}$ and $U_{100}, T=U_{50} / U_{100}$. Using Eq. (B1), we find the real part of the experimental wave number, defined as $k_{\mathrm{Le}}=\operatorname{Re}\left\{\ln (T) / i \Delta_{x}\right\}$, with $\Delta x=50 \mathrm{~cm}$. In Fig. 9(a) the light blue line displays the real part of $k_{\mathrm{Le}}$. We carry out a sweep over the experimental Young's modulus $E_{\mathrm{e}}$ within a set frequency range of 5 to $25 \mathrm{kHz}$, i.e., the spectrum of the Ricker source function, to find the $E_{\mathrm{e}}$ best minimizing the difference between the theoretical $k_{\mathrm{Lt}}$ and the

\section{Longitudinal wave number}

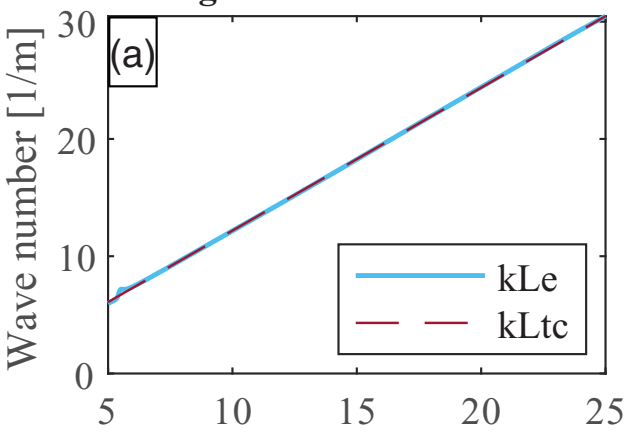

Flexural wave number

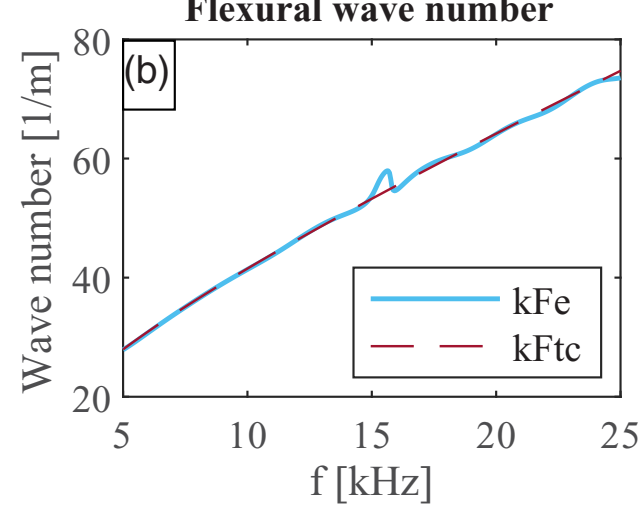

FIG. 9. Comparing the (a) longitudinal experimental $k_{\mathrm{Le}}$ and corrected theoretical $k_{\mathrm{Ltc}}$ wave numbers and (b) flexural experimental $k_{\mathrm{Fe}}$ and corrected theoretical $k_{\mathrm{Ftc}}$ wave numbers in the range from 5 to $25 \mathrm{kHz}$.

observed experimental wave number $k_{\mathrm{Le}}$. This provides us with a corrected theoretical wave number $k_{\mathrm{Ltc}}$, as depicted by the dashed dark red line in Fig. 9 and the experimental Young's modulus $E_{\mathrm{e}}=72.4 \mathrm{GPa}$. The corrected theoretical wave number $k_{\mathrm{Ltc}}$ is then used for redatuming any longitudinal waves within the aluminum beam.

When determining the experimental flexural wave number, we follow the same procedure. Using the Timoshenko beam theory [31], the dispersion relation of flexural waves is

$$
\begin{aligned}
k_{\mathrm{Ft}}= & \left\{\frac{1}{2}\left[\left(\frac{1}{C_{s}}\right)^{2}+\left(\frac{C_{r}}{C_{b}}\right)^{2}\right] \omega^{2}\right. \\
& \left.+\sqrt{\frac{\omega^{2}}{C_{b}^{2}}+\frac{1}{4}\left[\left(\frac{1}{C_{s}}\right)^{2}-\left(\frac{C_{r}}{C_{b}}\right)^{2}\right]^{2} \omega^{4}}\right\}^{1 / 2} .
\end{aligned}
$$

Equation (B2) includes the bending stiffness $C_{b}$, shear stiffness $C_{s}$, and rotational effects $C_{r}$ :

$$
C_{b}=\sqrt{\frac{E I}{\rho A}}, \quad C_{s}=\sqrt{\frac{G A \kappa}{\rho A}}, \quad C_{r}=\sqrt{\frac{\rho I}{\rho A}} .
$$

The cross section of the beam is $A=0.03^{2} \mathrm{~cm}^{2}$ and the Timoshenko shear coefficient is $\kappa=5 / 6$. The theoretical, flexural wave number $k_{\mathrm{Ft}}$ is found using an initial guess of the shear modulus, $G_{\mathrm{t}}=25 \mathrm{GPa}$. As for the longitudinal wave number, we choose the frequency range of $5-25 \mathrm{kHz}$ to perform a linear regression, minimizing the difference 
between the theoretical $k_{\mathrm{Ft}}$ and experimental $k_{\mathrm{Fe}}$ flexural wave numbers by finding the optimal, experimental value of the shear modulus $G_{\mathrm{e}}=27.6 \mathrm{GPa}$ [see Fig. 9(b)]. The determined corrected, theoretical flexural wave number $k_{\mathrm{Ftc}}$ is then used to redatum any flexural waves within the aluminum beam.

\section{APPENDIX C: EXPERIMENTALLY APPLYING THE IBC}

The workflow to design the wave field to be applied at the IBC source is the same for either pure longitudinal or flexural excitation, as well as for simultaneous excitation of the two. First, we excite the desired wave type at the SRC side. We measure the wave field $u(x, t)$ at two positions along the beam, $u\left(x_{1}, t\right)$ and $u\left(x_{2}, t\right)$, and apply the wave field separation algorithm (see Appendix A). Once the leftgoing wave forms $l\left(x_{2}, t\right)$ have been identified, the first reflected arrival $l\left(x_{2}, t\right)_{\text {refl }}$ is windowed and backward propagated to its point of reflection, $l\left(x_{\mathrm{IBC}}, t\right)_{\mathrm{refl}}$, the IBC end of the beam, using either the longitudinal or flexural experimental wave number (see Appendix B). To effectively cancel this reflection, the IBC source must inject the windowed and redatumed wave field with opposite polarity $-l\left(x_{\mathrm{IBC}}, t\right)_{\text {refl }}$. Hence, we must determine the voltage signal to apply to the IBC piezoelectric actuator such that the desired displacement $-l\left(x_{\mathrm{IBC}}, t\right)_{\mathrm{refl}}$ is induced at the IBC end of the beam. To this end, we excite the IBC source with a Ricker wavelet of central frequency $10 \mathrm{kHz}$ and measure the wave field at the middle of the aluminum beam. The first arriving wave form is isolated and back propagated to the IBC end. By comparing this signal to the original source signal, the inverse impulse response $h^{-1}(t)$ of the piezoelectric actuator is found. This process must be repeated for all three excitation directions of the piezoelectric actuator. Finally, the wave field $-l\left(x_{\mathrm{IBC}}, t\right)_{\mathrm{refl}}$ is convolved with the source transfer function $h^{-1}(t)$ to determine the wave form applied to the IBC piezoelectric actuator, $u^{\mathrm{IBC}}=-l\left(x_{\mathrm{IBC}}, t\right)_{\mathrm{reff}} *$ $h^{-1}(t)$. If both the SRC and IBC are actuated simultaneously, the reflection at the IBC boundary is effectively canceled.
[1] M. Vasmel, J. O. A. Robertsson, D.-J. van Manen, and A. Curtis, Immersive experimentation in a wave propagation laboratory, J. Acoust. Soc. Am. 134, EL492 (2013).

[2] D.-J. van Manen, J. O. A. Robertsson, and A. Curtis, Exact wave field simulation for finite-volume scattering problems, J. Acoust. Soc. Am. 122, EL115 (2007).

[3] T. S. Becker, D. J. van Manen, C. M. Donahue, C. Bärlocher, N. Börsing, F. Broggini, T. Haag, J. O. A. Robertsson, D. R. Schmidt, S. A. Greenhalgh, and T. E. Blum, Immersive Wave Propagation Experimentation: Physical Implementation and One-Dimensional Acoustic Results, Phys. Rev. X 8, 031011 (2018).

[4] M. Fink, From Loschmidt daemons to time-reversed waves, Philos. Trans. R. Soc. London A 374, 20150156 (2016).

[5] K. Wapenaar, J. Thorbecke, J. van der Neut, F. Broggini, E. Slob, and R. Snieder, Marchenko imaging, Geophysics 79, WA39 (2014).

[6] A. Fichtner, Full seismic waveform inversion for structural and source parameters, Ph.D. thesis, LMU Munich (unpublished).

[7] G. A. Gist, Interpreting laboratory velocity measurements in partially gas-saturated rocks, Geophysics 59, 1100 (1994).

[8] T. Cadoret and D. Marion, Influence of frequency and fluid distribution on elastic wave velocities in partially saturated limestones, J. Geophys. Res. 100, 9789 (1995).

[9] M. L. Batzle, D.-H. Han, and R. Hofmann, Fluid mobility and frequency-dependent seismic velocity-Direct measurements, Geophysics 71, N1 (2006).

[10] S. A. Cummer, J. Christensen, and A. Alu, Controlling sound with acoustic metamaterials, Nat. Rev. Mater. 1, 16001 (2016).

[11] J. Christensen, M. Kadic, O. Kraft, and M. Wegener, Vibrant times for mechanical metamaterials, MRS Commun. 5, 453 (2015).

[12] R. Fleury, D. L. Sounas, and A. Alu, Parity-time symmetry in acoustics: Theory, devices, and potential applications, IEEE J. Sel. Top. Quantum Electron. 22, 121 (2016).

[13] D.-J. van Manen, M. Vasmel, S. Greenhalgh, and J. O. A. Robertsson, Broadband cloaking and holography with exact boundary conditions, J. Acoust. Soc. Am. 137, EL415 (2015).

[14] N. Börsing, T. S. Becker, A. Curtis, D.-J. van Manen, T. Haag, and J. O. A. Robertsson, Cloaking and Holography Experiments using Immersive Boundary Conditions, Phys. Rev. Appl. 12, 024011 (2019).

[15] L. Ting and M. Miksis, Exact boundary conditions for scattering problems, J. Acoust. Soc. Am. 80, 1825 (1986).

[16] F. Broggini, M. Vasmel, J. O. A. Robertsson, and D.-J. van Manen, Immersive boundary conditions: Theory, implementation, and examples, Geophysics 82, T97 (2017).

[17] M. L. Vasmel, Immersive boundary conditions for seismic wave propagation, Ph.D. thesis, ETH Zurich, 2016 (unpublished).

[18] K. Aki and P. Richards, Quantitative Seismology (University Science Books, New York, 2002).

[19] H. R. Thomsen, D.-J. van Manen, and J. O. Robertsson, Exact wavefield separation on an elastic-free surface with sharp corners, in SEG Technical Program Expanded Abstracts 2018 (Society of Exploration Geophysicists, Tulsa, 2018) pp. 50175021.

[20] COMSOL AB, COMSOL Multiphysics v. 5.4, 2019, https: //www.comsol.com/.

[21] PI Ceramic GmbH, PICA Shear Actuators, 2018, https://www. piceramic.com/en/

[22] PiezoDrive, PD32-32 Channel Piezo Driver, 2019, https:// www.piezodrive.com/drivers/pd32-32-channel-piezo-driver/.

[23] Polytec GmbH, PSV-500-3D Scanning Vibrometer, 2019, https://www.polytec.com/us/vibrometry/products/full-fieldvibrometers/psv-500-3d-scanning-vibrometer/.

[24] L. Sønneland, L. E. Berg, P. Eidsvig, A. Haugen, B. Fotland, and J. Vestby, 2-D deghosting using vertical receiver arrays, in SEG Technical Program Expanded Abstracts 1986, Vol. 4 (Society of Exploration Geophysicists, Tulsa, 1986) pp. 516519.

[25] See Supplemental Material at http://link.aps.org/supplemental/ 10.1103/PhysRevResearch.1.033203 for corresponding videos and their description. 
[26] R. J. Marks II, Introduction to Shannon Sampling and Interpolation Theory (Springer-Verlag, New York, 1991).

[27] X. Li, J. Robertsson, A. Curtis, and D.-J. van Manen, Compensating for source directivity in immersive wave experimentation, J. Acoust. Soc. Am. 146, 3141 (2019).

[28] J. O. Robertsson, I. Moore, M. Vassallo, K. Özdemir, D.J. van Manen, and A. Özbek, On the use of multicomponent streamer recordings for reconstruction of pressure wavefields in the crossline direction, Geophysics 73, A45 (2008).
[29] M. Vassallo, A. Özbek, K. Özdemir, and K. Eggenberger, Crossline wavefield reconstruction from multi-component streamer data: Multichannel interpolation by matching pursuit (MIMAP) using pressure and its crossline gradient, Geophysics 75, WB53 (2010).

[30] C. P. Wapenaarand G. C. Haimé, Elastic extrapolation of primary seismic p- and s-waves, Geophys. Prospect. 38, 23 (1990).

[31] C. Mei and B. R. Mace, Wave reflection and transmission in Timoshenko beams and wave analysis of Timoshenko beam structures, J. Vib. Acoust 127, 382 (2005). 